\title{
Primary small cell carcinoma of the urinary bladder: a 10-year retrospective review of treatment and survival at a single institution.
}

\author{
Xue-Jun Liu, Cheng Liu, Duo Liu, Dong-Wei Yao* \\ Department of Urology, the Second People's Hospital of Lianyungang, Lianyungang, Jiangsu, PR China
}

\begin{abstract}
Objective: Primary small cell carcinoma of the urinary bladder (SCBC) is a rare condition. SCBC accounts for less than $1 \%$ of all urinary bladder tumors. The purpose of this study is to report the clinical experience, clinical features, treatment modalities and overall survival of patients diagnosed with SCBC.

Patients and methods: There were 12 patients who were diagnosed with SCBC between January 2002 and December 2012 at our institution. The features of the patients, including age, gender, smoking history, presenting symptoms, tumor size, tumor locations, treatment modalities, stages, pathology appearance, recurrence and overall survival, were retrospectively analyzed.

Results: Twelve patients were diagnosed with SCBC (male: female ratio, 3:1; mean age, 69.8 $\pm 7.1 \mathrm{y}$; mean follow-up, $18.3 \pm 5.6$ months). Nine of these patients had a history of smoking. Eleven patients presented with gross hematuria, and the other patient presented with urinary frequency. Only one patient had stage I disease; eight patients had stage III disease, and the other three patients had stage IV disease. The mean tumor size was $4.62 \pm 1.33 \mathrm{~cm}$ in diameter. Nine patients were treated with surgery (six patients underwent radical cystectomy, and three patients underwent TUR-BT); the other three patients received sequential chemoradiotherapy because of the presence of lymph node or distant metastasis at the time of the initial diagnosis. Overall, eight patients were treated with chemotherapy. At the time of data analysis, only one patient was alive. The median survival time of all patients was 14 months. The median survival time was 19 months for patients receiving chemotherapy compared to 12.5 months for patients who did not receive chemotherapy.

Conclusion: Although many efforts have been made to treat SCBC, there is still no standard strategy for treatment. Despite the high response rate to chemotherapy, the overall prognosis is poor. SCBC is an aggressive disease with a propensity for early metastasis. Prospective multi-institutional randomized studies are needed to establish the best treatment modalities. New molecular markers for early diagnosis and novel targeted therapies may play important roles in the future.
\end{abstract}

Keywords: Small cell carcinoma, Bladder cancer, Treatment, Prognosis.

Accepted on February 06, 2018

\section{Introduction}

SCBC is very uncommon, accounting for less than $1 \%$ of all urinary bladder carcinomas [1]. This disease was first described in 1981 by Cramer et al. [2]. Since then, nearly 250 cases have been reported in the literature [3]. Although rare, $\mathrm{SCBC}$ is an aggressive malignant tumor with a poor prognosis, as many patients have progressed to advanced stages and exhibit early metastasis by the time that they are diagnosed. This disease affects mostly elderly male patients with a history of cigarette smoking. The most common symptom of SCBC is gross hematuria; a few patients present with urinary frequency, dysuria or pain. Paraneoplastic syndrome is uncommon. However, because of the relative rarity of SCBC, there is no established treatment strategy for managing this disease. In previously published studies, different treatments, including surgery, radiotherapy (RT), chemotherapy (CT) and combinations of treatments were used. We reported our clinical experience of treating 12 patients with SCBC at our hospital between 2002 and 2012 .

\section{Patients and Methods}

Each patient provided written informed consent, and our hospital ethical review board approved the study. We retrospectively analyzed data from all of the patients diagnosed with bladder carcinoma from January 2002 to December 2012 at our hospital. A total of 1963 patients with bladder tumors were reviewed, and there were 12 patients who had been diagnosed with SCBC (excluding tumors from other sites with metastasis to the bladder). SCBC accounted for approximately $0.61 \%$ of all bladder tumors at our institution. We focused on age, smoking history, gender, presented symptoms, tumor size, tumor locations, treatment modalities, stages, pathology 
appearance, recurrence and overall survival. Radical cystectomy (RC) was performed in six patients. The RC surgical procedure was complex and consisted of several steps, including pelvic lymph node dissection (PLND), the RC itself, and urinary diversion. In our study, there were eight patients who received cisplatin-based chemotherapy; all patients signed consent to receive chemotherapy preoperatively. Chemotherapy regimens varied according to the components of the tumor. SCBC patients with non-small-cell carcinomatous components received four cycles of alternating chemotherapy. Ifosfamide $2,000 \mathrm{mg} / \mathrm{m}^{2}$ was infused over $3 \mathrm{~h}$ daily on days 1 through 4. Doxorubicin $25 \mathrm{mg} / \mathrm{m}^{2}$ was infused daily on days 1 through 3. Patients received the EP regimen (etoposide plus cisplatin) at 3 -week intervals. Etoposide $100 \mathrm{mg} / \mathrm{m}^{2}$ was infused over $2 \mathrm{~h}$ daily on days 1 through 5 ; cisplatin $40 \mathrm{mg} / \mathrm{d}$ was infused in $1 \mathrm{~L}$ of normal saline with mannitol $20 \mathrm{~g}$ daily on days 1 through 3 . Each regimen was repeated every 6 weeks. Pure SCBC patients received the EP regimen, which was repeated every 3 weeks. All of the patients received four cycles unless serious adverse reactions occurred. Two-phase radiotherapy was used; the dose was 45 Gy to the pelvis followed by a boost of 15 Gy to the bladder (total tumor dose 60 Gy).

\section{Statistical analysis}

All statistical analyses were conducted by using SPSS version 20.0 statistical software; all data were expressed as $\overline{\mathrm{x}} \pm \mathrm{s}$ Survival curves were plotted using the Kaplan-Meier method.

\section{Results}

The general features of the patients are listed in Table 1. Patients with SCBC were predominantly male (male: female ratio 3:1), and the mean age at diagnosis was $69.8 \pm 7.1 \mathrm{y}$ old. Nine patients $(75 \%)$ had a history of cigarette smoking. Eleven patients presented with gross hematuria, and the other patient presented with urinary frequency. According to the 2002 TNM classification of urinary bladder cancer, patients were staged based on computed tomography (CT), chest x-ray, bone scan and postoperative pathological results [4]. Only one patient was diagnosed with stage I disease, eight patients had stage III disease, and the other three had stage IV disease (Table 1).

Table 1. Features of the patients with SCBC $(n=12)$.

\begin{tabular}{ll}
\hline Features & $\mathbf{N}(\%)$ \\
\hline Age $(\mathrm{y})$ & $1(8.33)$ \\
\hline$<60$ & $7(58.33)$ \\
\hline $60-70$ & $4(33.33)$ \\
\hline$>70$ & \\
\hline Smoking history & $9(75)$ \\
\hline Smoker & $3(25)$ \\
\hline Non-smoker & \\
\hline Gender &
\end{tabular}

\begin{tabular}{ll}
\hline Male & $9(75)$ \\
\hline Female & $3(25)$ \\
\hline Present symptoms & $11(91.67)$ \\
\hline Gross hematuria & $1(8.33)$ \\
\hline Urinary frequency & \\
\hline Stage & $1(8.33)$ \\
\hline I & $0(0)$ \\
\hline II & $8(66.67)$ \\
\hline III & $3(25)$ \\
\hline IV &
\end{tabular}

Treatments of SCBC are shown in Table 2. Six patients underwent radical cystectomy, two of whom received neoadjuvant chemotherapy and two received adjuvant chemotherapy. Three patients were treated with transurethral resection of the bladder tumor (TUR-BT), and one of them received adjuvant chemotherapy. Three patients received sequential chemoradiotherapy because of the presence of lymph node metastasis or distant metastasis at the time of diagnosis. In all, 8 patients were treated with chemotherapy, and two of them received cisplatin-based regimens as described before. Prophylactic cranial irradiation (PCI) was not performed on these patients.

Table 2. The details and outcomes of the patients with SCBC.

\begin{tabular}{|c|c|c|c|c|}
\hline Patient & Stage & Treatment & Relapse/death & Outcomes (m) \\
\hline 1 & 1 & TUR-BT & Lung & Dead (11) \\
\hline 2 & III & TUR-BT+CT & & Alive (12) \\
\hline 3 & III & TUR-BT & Liver & Dead (9) \\
\hline 4 & III & Neoadjuvant+RC & Lung & Dead (30 \\
\hline 5 & III & Neoadjuvant+RC & Bone & Dead (41) \\
\hline 6 & III & RC+adjuvant & Brain & Dead (26) \\
\hline 7 & III & $\mathrm{RC}+$ adjuvant & Pelvis & Dead (19) \\
\hline 8 & III & $\mathrm{RC}$ & Lung & Dead (16) \\
\hline 9 & III & $\mathrm{RC}$ & Bone & Dead (14) \\
\hline 10 & IV & Chemoradiotherapy & Lung infection & Dead (10) \\
\hline 11 & IV & Chemoradiotherapy & Neutropenia & Dead (12) \\
\hline 12 & IV & Chemoradiotherapy & Respiratory failure & Dead (7) \\
\hline
\end{tabular}

Abbreviations: TUR-BT: Transurethral Resection of the Bladder Tumor; CT: Chemotherapy; RC: Radical Cystectomy.

The tumor characteristics are shown in Table 3. The mean tumor size was $4.62 \pm 1.33 \mathrm{~cm}$ in diameter. Eight tumors were located on the lateral wall; two tumors were located in the fundus, one in the trigone, and one in the dome of the bladder. 
Primary small cell carcinoma of the urinary bladder: a 10-year retrospective review of treatment and survival at a single institution

Our study identified five patients with pure SCBC, which accounted for $41.6 \%$ of the total patient population.

Table 3. The tumor characteristics of the patients with SCBC.

\begin{tabular}{ll}
\hline Tumor characteristics & N (\%) \\
\hline Tumor size (diameter) & $5(41.67)$ \\
\hline$<4 \mathrm{~cm}$ & $3(25)$ \\
\hline $4-6 \mathrm{~cm}$ & $4(33.33)$ \\
\hline$>6 \mathrm{~cm}$ & \\
\hline Main tumor location & $1(8.33)$ \\
\hline Dome & $4(33.33)$ \\
\hline Left lateral & $4(33.33)$ \\
\hline Right lateral & $1(8.33)$ \\
\hline Trigone & $2(16.67)$ \\
\hline Fundus & \\
\hline Tumor components & $5(41.67)$ \\
\hline Pure SCC & $7(58.33)$ \\
\hline SCC with non-small-cell carcinomatous components
\end{tabular}

The average follow-up time was $18.3 \pm 5.6$ months; at the time of this analysis, only one patient was alive. The overall median survival time was 14 months (Figure 1).

Regardless of the clinical stage, the median survival time was 19 months for patients receiving chemotherapy, versus 12.5 months for patients who had not received chemotherapy (Figure 2).

Tumors were consisted of small cells with a lack of cytoplasm, which also had uniform nucleus size, fine granular chromatin and insignificant nucleoli. Mitotic images were common (Figure 3).

\section{Discussion}

$\mathrm{SCBC}$ is a rare type of bladder cancer that only accounts for less than $1 \%$ of all urinary bladder cancers. SCBC is an aggressive malignant tumor that is detected in advanced stages and patients diagnosed with SCBC have a poor prognosis. Although rare, a dramatic increase in the incidence of SCBC in the past $15 \mathrm{y}$ has been reported by Koay et al. [1].

The incidence has significantly increased from 0.05 cases to 0.14 cases per 100,000 people from 1991 to 2005 , which might be associated with population aging [1].

SCBC normally affects older male patients with a history of cigarette smoking. According to previous studies, most patients were in their sixth to seventh decade of life [5-8].

The etiology of SCBC is still uncertain. Different theories have been postulated; the most widely accepted theory is that SCBC originates from a multi-potent stem cell that has the ability to differentiate into various cell types.

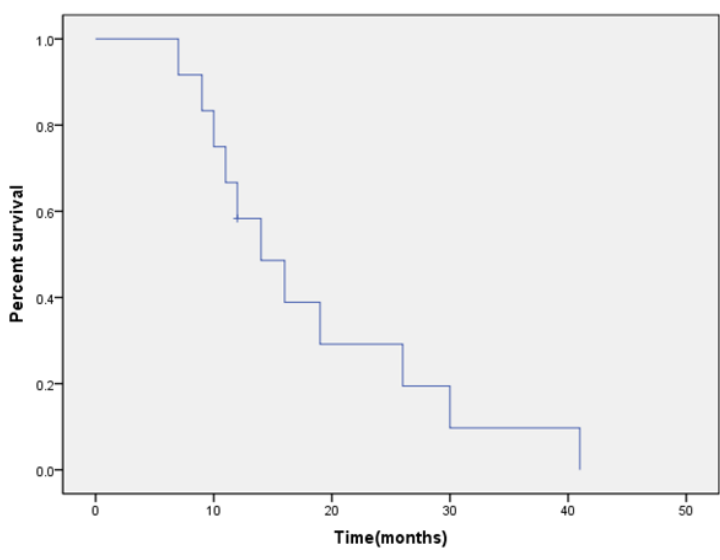

Figure 1. Kaplan-Meier survival rates for patients with SCBC.

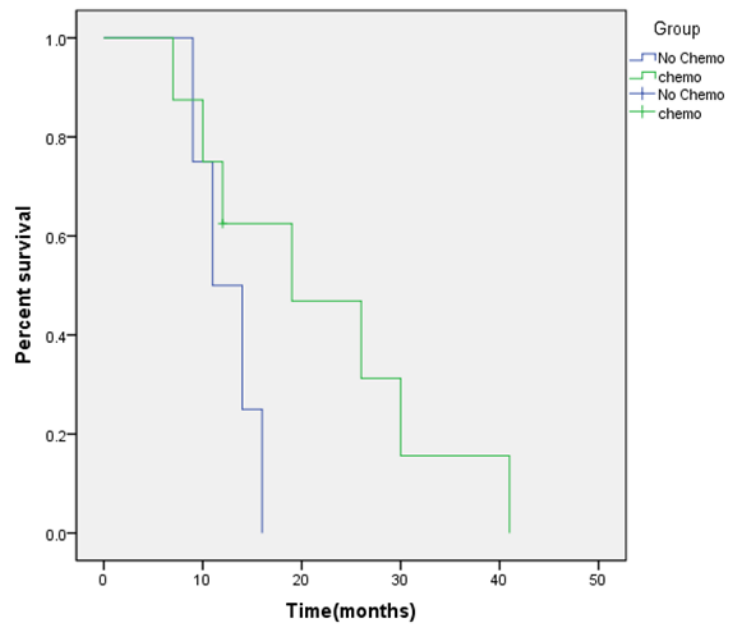

Figure 2. Kaplan-Meier survival curves for SCBC patients with or without chemotherapy.

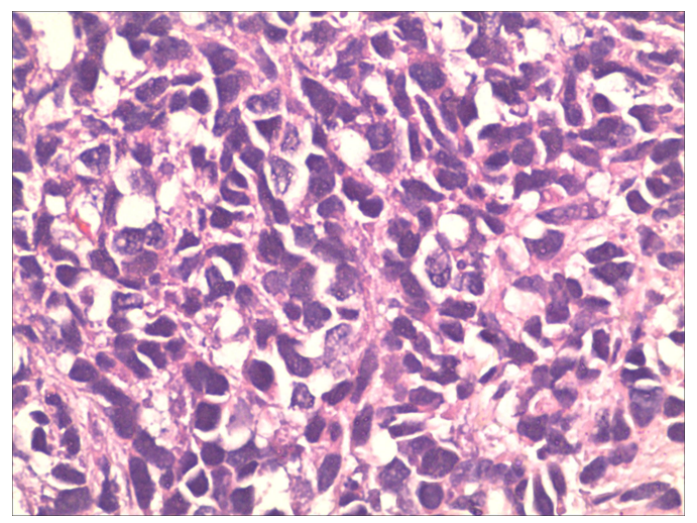

Figure 3. Pathologic characteristics: Tumors were consisted of small cells with a lack of cytoplasm, which also had uniform nucleus size, fine granular chromatin and insignificant nucleoli. Mitotic images were common $(H E \times 400)$.

This theory was proposed due to the coexistence of SCBC with other types of bladder cancer such as urothelial carcinoma, adenocarcinoma, or squamous cell carcinoma within the same tumor [9-11]. Further support for this theory derives from the recent report by Cheng et al., which demonstrated an identical 
pattern of allelic loss of coexistent urothelial and small cell counterparts, suggesting a common cell of origin [12]. Other hypotheses include the derivation of SCBC from the amine precursor uptake and decarboxylation system, and metaplasia from other high-grade malignancies $[2,5]$.

According to the previous study by Church et al., cigarette smoking was a risky factor of SCBC [13]. The most common presentation was gross hematuria, which was similar to transitional cell carcinoma (TCC) [5,9,14-16]. Dysuria, pelvic pain, local irritation, urinary obstruction and urinary infections were other common symptoms. Some patients may also present with systemic symptoms including anorexia, fatigue and weight loss. However, paraneoplastic syndromes including Cushing syndrome, hypercalcemia and hypophosphatemia were less commonly observed [9,15-19]. Most of the tumors were located on the lateral wall of the bladder and were less common in the fundus, trigone, anterior wall, and dome of the bladder $[1,20]$.

The diagnosis of SCBC was based on the WHO classification system (2004), which was also used for the diagnosis of lung small cell carcinoma (SCLC) [21]. Under microscopic examination, the cancer cells stained with hematoxylin and eosin have scant cytoplasm with few organelles. Tumors are composed of small or intermediate-sized malignant cells in nests or sheets with marked hypercellularity, necrosis and mitosis. Individual cells have rounded to oval overlapping nuclei with evenly distributed salt-and-pepper-like chromatin [21]. The deposition of basophilic material around blood vessels (the Azzopardi phenomenon) is also observed in many cases. Immunohistochemistry plays an important role in the diagnosis of SCBC. Most of the SCBC express neural markers, of which neuron-specific enolase is the most common [22,23]. Chromogranin A and synaptophysin positive cells are detected in half of the cases. Cytokeratin (CAM 5.2) is expressed in $25 \%$ of SCBC, and the expression pattern in SCBC is different from the expression pattern in high grade urothelial tumors [24]. Immunocytochemical studies are useful for distinguishing SCBC from other tumors such as malignant lymphoma, lymphoepithelioma-like carcinoma, Merkel cell carcinoma and poorly differentiated TCC. Chromogranin A is detectable in $50 \%$ of SCBC samples and only $5 \%$ of urothelial carcinoma samples. CD44v6 is positive in only $7 \%$ of SCBC samples and $60 \%$ of urothelial tumors. Cytokeratin 20 expression is positive in $46-73 \%$ of urothelial tumors and is absent in SCBC [25].

In the majority of patients (38\% to $70 \%)$, SCBC coexisted with non-small-cell carcinomatous components including urothelial carcinoma, adenocarcinoma, squamous cell carcinoma or sarcomatoid carcinoma [26]. Pure SCBC is uncommon [21]. According to a previous study by Hou et al., SCBC was most often present with UC; UC was detected in $61.11 \%$ of patients with SCBC [27]. Cramer et al. suggested that SCBC might originate from metaplastic urothelium [2]. Other studies proposed that SCBC originated from a multi-potential stem cell with the ability to differentiate into various cell types. These hypotheses might explain the coexistence of SCBC with UC [28].
Staging of SCBC is based on the TNM staging system, which is used for UC. Unlike urothelial cancers, when the diagnosis is confirmed, most patients with SCBC are diagnosed with an advanced stage of the disease, even though some patients are initially diagnosed with organ confined disease [29]. This disease progresses quickly and becomes metastatic $[1,30]$. A clinically relevant two-stage system of limited (T1-4N0-1M0) and extensive disease (TxNxM1 or TxN2-3M0) is widely used to determine prognosis and treatment. Limited disease (LD) is classified as a disease localized to an area that could be included in 1 radiation port or surgical field. Extensive disease (ED) refers to any disease beyond LD [1].

The optimum therapy for SCBC is difficult to define. Because it is a rare tumor, there are no standard guidelines for treatment and most of the treatment decisions are based on retrospective studies from a single center. The management of SCBC involves a multimodal approach including chemotherapy, chemo- radiotherapy, and surgery with or without chemotherapy. Chemotherapy is the mainstay of treatment and improves overall survival [31,32]. Clinical stage and performance status are the determining factors that guide treatment decisions in patients with SCBC. Surgeries, including radical cystectomy, partial cystectomy, and TUR-BT, are commonly performed for the LD form of SCBC. TUR-BT alone is probably inadequate for LD patients due to high recurrence rates and a short mean survival of only 3 to 6 months [23,33]. TUR-BT is only performed when the general condition of the patient is not suitable for other treatment modalities or when the patient requests bladder-sparing surgery.

Radical cystectomy alone may not be curative in the majority of cases. It might be adequate for patients with early stage disease, as described in the studies from the Mayo clinic in which eight patients were diagnosed with stage II SCBC. In this study, six patients survived for at least $5 \mathrm{y}$, one patient had local recurrence and one patient developed metastatic disease less than one year after the surgery [5]. However, as most patients with SCBC present with advanced stage disease at the time of diagnosis, surgery without chemotherapy may be inadequate to treat SCBC. In a review by Cheng et al., there was no significant difference in survival between patients who underwent cystectomy and those who did not (5-y survival, $15 \%$ vs. $18 \%, \mathrm{P}=0.65)$ [14]. According to a recent report from the MD Anderson Cancer Center, 52\% of patients who underwent cystectomy had a better survival, and neoadjuvant chemotherapy administered before surgery played an important role in improving overall survival and in the pathologic downgrading of the tumor $[8,34]$. Half of our patients received radical cystectomy, and four received neoadjuvant or adjuvant chemotherapy (Table 2).

The most common regimen performed for SCBC is platinumbased chemotherapy (etoposide and cisplatin), which appears to improve overall survival. Mackey et al. reported that cisplatin-based chemotherapy was the only type of chemotherapy associated with better overall survival according to a multivariable analysis [31]. According to the study by Bex 


\section{single institution}

et al., there is a significant difference in survival apparently between SCBC patients who received chemotherapy and those who did not, regardless of the tumor stage. The median duration of survival of patients who received chemotherapy was 15 months, while the length of survival of patients without chemotherapy was only 4 months $(\mathrm{P}=0.003)$ [8]. In another published study, patients who received neoadjuvant chemotherapy had a significantly better 5-y survival rate than those who had undergone cystectomy alone (78\% vs. $36 \%$, $\mathrm{P}=0.026$ ) [35]. Quek et al. reported on a series of 20 patients who had undergone cystectomy. Patients treated with neoadjuvant or adjuvant chemotherapy had significantly better overall and tumor-free survival [7]. In our study, eight patients received cisplatin-based chemotherapy. All of the patients received four cycles unless serious adverse reactions occurred. The median survival time was 14 months, but the patients who had received chemotherapy had improved outcomes, with a median survival of 19 months compared with 12.5 months in the patients who had not received chemotherapy (Figures 1 and 2 ). Despite the rarity of this cancer and a lack of randomized controlled studies, the available data strongly suggests a significant survival advantage with chemotherapy.

Chemoradiotherapy has been shown to be effective in the treatment of SCLC and has been used in many studies for SCBC [36]. Oblon et al. first described chemotherapy combined with radiotherapy for SCBC [37]. In a retrospective study by Bex et al., 17 patients with LD received sequential chemo-radiotherapy followed by TUR-BT, $88 \%$ of patients had a complete response, and the median overall survival was 32.5 months [8]. Bastus et al. reported on 5 patients with SCBC treated with platinum-based chemotherapy and radiotherapy; four patients had a complete response and only one exhibited local relapse. The patients had a disease-free survival of between 10 to 60 months [38]. Lohrisch et al. described a retrospective analysis of $10 \mathrm{LD}$ patients in whom the overall survival at 2 and 5 y was $70 \%$ and $44 \%$, respectively. Chemoradiotherapy has been shown to improve survival, and when combined with transurethral resection, could be used as a trimodal approach for patients who wish to avoid or delay radical cystectomy [15]. However, these patients accepted that chemo- radiotherapy with bladder preservation may increase the risk for the development of urothelial cancers, although it is uncommon [15].

Patients with metastasis have a poor prognosis, with a median survival of approximately 7 to 13 months [34,39]. A cisplatinbased chemotherapy regimen is generally considered to be the best choice $[5,15,36]$. The incidence of brain metastases in patients with SCLC is approximately $50-60 \%$ over $2 \mathrm{y}$. Prophylactic cranial irradiation (PCI) is the established treatment for patients with SCLC who have a complete response to therapy; PCI has been shown to prolong diseasefree survival and to improve overall survival [22]. Nevertheless, data on the use of PCI to prevent brain metastases in SCBC is limited, and the role of PCI is unclear.

SCBC is a rare and aggressive tumor; patients often present with an advanced stage of the disease and have a poor prognosis. SCBC mostly affects older men with a history of smoking. Because SCBC is rare, there are no standard guidelines for the management of patients. Chemotherapy is the mainstay of treatment and has demonstrated survival benefits. Based on previous studies, we recommend a combined modality approach for SCBC. Patients with LD who are eligible for surgery may experience better outcomes with neoadjuvant chemotherapy followed by radical cystectomy. For patients not eligible for surgery, sequential chemoradiotherapy might improve overall survival. Palliative chemotherapy should be offered to patients with extensive disease.

\section{References}

1. Koay EJ, Teh BS, Paulino AC, Butler EB. A surveillance, epidemiology, and end results analysis of small cell carcinoma of the bladder. Cancer 2011; 117: 5325-5333.

2. Cramer SF, Aikawa M, Cebelin M. Neurosecretory granules in small cell invasive carcinoma of the urinary bladder. Cancer 1981; 47: 724-730.

3. Wang X, MacLennan GT, Lopez-Beltran A, Cheng L. Small cell carcinoma of the urinary bladder-histogenesis, genetics, diagnosis, biomarkers, treatment, and prognosis. Appl Immunohistochem Mol Morphol 2007; 15: 8-18.

4. Oosterlinck W, Lobel B, Jakse G, Malmström PU, Stöckle M, Sternberg C. Guidelines on bladder cancer. Eur Urol 2002; 41: 105-112.

5. Choong NW, Quevedo JF, Kaur JS. Small cell carcinoma of the urinary bladder. Cancer 2005; 103: 1172-1178.

6. Montie JE. Small cell carcinoma of the urinary bladder. A clinicopathologic analysis of 64 patients. J Urol 2005; 173: 1920-1921.

7. Quek ML, Nichols PW, Yamzon J, Daneshmand S, Miranda G, Cai J, Groshen S, Stein JP, Skinner DG. Radical cystectomy for primary neuroendocrine tumors of the bladder: the University of Southern California experience. J Urol 2005; 174: 93-96.

8. Bex A, Nieuwenhuijzen JA, Kerst M, Pos F, van Boven H, Meinhardt W, and Horenblas S. Small cell carcinoma of bladder: a single-center prospective study of 25 cases treated in analogy to small cell lung cancer. Urology 2005; 65: 295-299.

9. Blomjos CE, Vos W, de Voogt HJ, Valk PVD, Meijer CJ. Small cell carcinoma of the urinary bladder. A clinicopathologic, morphometric, immunohistochemical, and ultrastructural study of 18 cases. Cancer 1989; 64: 1347-1357.

10. Holmang S, Borghede G, Johansson SL. Primary small cell carcinoma of the bladder: a report of 25 cases. J Urol 1995; 153: 1820-1822.

11. Abbas F, Civantos F, Benedetto P, Soloway MS. Small cell carcinoma of the bladder and prostate. Urology 1995; 46: 617-630.

12. Cheng L, Jones TD, McCarthy RP, Eble JN, Wang M, MacLennan GT, Lopez-Beltran A, Yang XJ, Koch MO, Zhang S. Molecular genetic evidence for a common clonal 
origin of urinary bladder small cell carcinoma and coexisting urothelial carcinoma. Am J Pathol 2005; 166: 1533-1539.

13. Church DN, Bahl A. Clinical review-small cell carcinoma of the bladder. Cancer Treat Rev 2006; 32: 588-593.

14. Cheng L, Pan CX, Yang XJ, Lopez-Beltran A, MacLennan GT, Lin H, Kuzel TM, Papavero V, Tretiakova M, Nigro K, Koch MO, Eble JN. Small cell carcinoma of the urinary bladder: a clinicopathologic analysis of 64 patients. Cancer 2004; 101: 957-962.

15. Lohrisch C, Murray N, Pickles T, Sullivan L. Small cell carcinoma of the bladder. Cancer 1999; 86: 2346-2352.

16. Mangar SA, Logue JP, Shanks JH, Cooper RA, Cowan RA, Wylie JP. Small-cell carcinoma of the urinary bladder: 10year experience. Clin Oncol 2004; 16: 523-527.

17. Mills SE, Wolfe III JT, Weiss MA, Swanson PE, Wick MR, Fowler Jr JE, Young RH. Small cell undifferentiated carcinoma of the urinary bladder: a light-microscopic, immunocytochemical, and ultrastructural study of 12 cases. Am J Surg Pathol 1987; 11: 606-617.

18. Reyes CV, Soneru I. Small cell carcinoma of the urinary bladder with hypercalcemia. Cancer 1985; 56: 2530-2533.

19. Partanen S, Asikainen U. Oat cell carcinoma of the urinary bladder with ectopic adrenocorticotropic hormone production. Hum Pathol 1985; 16: 313-315.

20. Ismaili N, Arifi S, Flechon A, El Mesbahi O, Blay J, Droz J, Errihani H. Small cell cancer of the bladder: pathology, diagnosis, treatment and prognosis. Bulletin Du Cancer 2009; 96: 10030-10044.

21. Abrahams N, Moran C, Reyes A, Siefker-Radtke A, Ayala A. Small cell carcinoma of the bladder: a contemporary clinicopathological study of 51 cases. Histopathology 2005; 46: 57-63.

22. Aupérin A, Arriagada R, Pignon J-P, Le Péchoux C, Gregor A, Stephens RJ, Kristjansen PE, Johnson BE, Ueoka H, Wagner H. Prophylactic cranial irradiation for patients with small-cell lung cancer in complete remission. N Engl J Med 1999; 341: 476-484.

23. Trias I, Algaba F, Condom E, Espanol I, Segui J, Orsola I, Villavicencio H, Garcia Del Muro X. Small cell carcinoma of the urinary bladder. Presentation of 23 cases and review of 134 published cases. Eur Urol 2001; 39: 85-90.

24. Cheng C, Nicholson A, Lowe D, Kirby R. Oat cell carcinoma of urinary bladder. Urology 1992; 39: 504-507.

25. Iczkowski K, Shanks J, Allsbrook W, Lopez-Beltran A, Pantazis C, Collins T, Wetherington R, Bostwick D. Small cell carcinoma of urinary bladder is differentiated from urothelial carcinoma by chromogranin expression, absence of CD44 variant 6 expression, a unique pattern of cytokeratin expression, and more intense $\gamma$-enolase expression. Histopathology 1999; 35: 150-156.

26. Urrea YR, Epstein JI. Sarcomatoid carcinoma associated with small cell carcinoma of the urinary bladder: a series of 28 cases. Hum Pathol 2017; 67: 169-175.
27. Hou CP, Lin YH, Chen CL, Chang PL, Tsui KH. Clinical outcome of primary small cell carcinoma of the urinary bladder. Onco Targets Ther 2013; 6: 1179.

28. Terracciano L, Richter J, Tornillo L, Beffa L, Diener PA, Maurer R, Gasser TC, Moch H, Mihatsch MJ, Sauter G. Chromosomal imbalances in small cell carcinomas of the urinary bladder. J Pathol 1999; 189: 230-235.

29. Ghervan L, Zaharie A, Ene B, Elec FI. Small-cell carcinoma of the urinary bladder: where do we stand. Clujul Med 2017; 90: 13-17.

30. Chen Z, Liu Q, Chen R. Clinical analysis of small cell carcinoma of the bladder in Chinese: nine case reports and literature reviews. World J Surg Oncol 2017; 15: 33.

31. Mackey JR, Au HJ, Hugh J, Venner P. Genitourinary small cell carcinoma: determination of clinical and therapeutic factors associated with survival. J Urol 1998; 159: 1624-1629.

32. Moretto P, Wood L, Emmenegger U. Management of small cell carcinoma of the bladder: consensus guidelines from the Canadian Association of Genitourinary Medical Oncologists (CAGMO). Can Urol Assoc J 2013; 7: 44-56.

33. Helpap B. Morphology and therapeutic strategies for neuroendocrine tumors of the genitourinary tract. Cancer 2002; 95: 1415-1420.

34. Lynch SP, Shen Y, Kamat A, Grossman HB, Shah JB, Millikan RE, Dinney CP, Siefker-Radtke A. Neoadjuvant chemotherapy in small cell urothelial cancer improves pathologic downstaging and long-term outcomes: results from a retrospective study at the MD Anderson Cancer Center. Eur Urol 2013; 64: 307-313.

35. Siefker-Radtke AO, Dinney CP, Abrahams NA, Moran C, Shen Y, Pisters LL, Grossman HB, Swanson DA, Millikan RE. Evidence supporting preoperative chemotherapy for small cell carcinoma of the bladder: a retrospective review of the MD Anderson cancer experience. J Urol 2004; 172: 481-484.

36. Pignon J-P, Arriagada R, Ihde DC, Johnson DH, Perry MC, Souhami RL, Brodin O, Joss RA, Kies MS, Lebeau B. A meta-analysis of thoracic radiotherapy for small-cell lung cancer. N Engl J Med 1992; 327: 1618-1624.

37. Oblon DJ, Parsons JT, Zander DS, Wajsman Z. Bladder preservation and durable complete remission of small cell carcinoma of the bladder with systemic chemotherapy and adjuvant radiation therapy. Cancer 1993; 71: 2581-2584.

38. Bastus R, Caballero J, Gonzalez G, Borrat P, Casalots J, Gomez de Segura G, Ll M, Ristol J, Cirera L. Small cell carcinoma of the urinary bladder treated with chemotherapy and radiotherapy: results in five cases. Eur Urol 1999; 35: 323-326.

39. Ismaili N, Heudel PE, Elkarak F, Kaikani W, Bajard A, Ismaili M, Errihani H, Droz JP, Flechon A. Outcome of recurrent and metastatic small cell carcinoma of the bladder. BMC Urol 2009; 9: 4. 
Primary small cell carcinoma of the urinary bladder: a 10-year retrospective review of treatment and survival at a single institution

\section{*Correspondence to}

Dong-Wei Yao

Department of Urology

The Second People's Hospital of Lianyungang

Lianyungang

Jiangsu

PR China 